\title{
PROBING ASPECTS OF NONLINEAR CONDUCTION IN ELECTRORHEOLOGICAL SUSPENSIONS
}

\author{
P.J. RANKIN, Y.M. SHKEL, AND D.J KLINGENBERG \\ Department of Chemical Engineering and Rheology Research Center, University of \\ Wisconsin, 1415 Engineering Dr., Madison WI 53706, USA \\ E-mail: klingen@engr.wisc.edu \\ J.L. SHOHET \\ Department of Electrical and Computer Engineering, University of Wisconsin,1415 \\ Engineering Dr., Madison WI 53706, USA \\ E-mail: shohet@engr.wisc.edu
}

\begin{abstract}
Particle and electrode surfaces were altered to examine the effects on the electrorheological response of barium titanate/silicone oil suspensions. Unmodified suspensions exhibited nonlinear conduction. The dynamic yield stresses scaled as $E^{n}$, where $n<2$ at all electric field frequencies, and the current had substantial harmonic content, a hallmark of nonlinear conduction. Modifying the particle surfaces did not affect these nonlinear responses. Casting polymers on the electrode surfaces changed the responses. With polymer coatings, the dynamic yield stresses scaled as $E^{n}$, where $n \geq 2$ at some frequencies and increased with coating thickness. With coated electrodes, the current harmonic contents were significantly decreased. These results suggest that nonlinear conduction in ER suspensions is associated with charge injection at the electrode/liquid interface.
\end{abstract}

\section{Introduction}

A significant limitation to the development of electrorheological (ER) fluids is an incomplete understanding of the underlying mechanisms. The electrostatic polarization model, which attributes the ER effect to the field-induced polarization of the dispersed phase relative to the continuous phase, ${ }^{1}$ qualitatively explains most features. The model predicts that polarization results in the formation of particle chains that span the electrode gap. The force required to break these chains is directly proportional to the fluid's yield stress $\left(\tau_{o}\right)$. The Maxwell-Wagner model describes polarization arising from permittivity and conductivity differences between the dispersed and continuous phases. ${ }^{2}$ This model predicts that the yield stress is proportional to the square of the field strength $\left(\tau_{o} \propto E^{2}\right)$, as observed experimentally. ${ }^{3}$

Experimental data cannot always be described by the above model. At large field strengths, yield stresses often vary as $\tau_{o} \propto E^{n}$, where $n<2$, in contrast to the $E^{2}$ dependence observed at smaller fields. ${ }^{4,5}$ This nonlinear behavior at large field strengths is accompanied by an increase in suspension conductivity. These nonlinear effects are detrimental to ER technology because they simultaneously diminish the potential rheological response, as well as increase the power demand.

Nonlinear conduction is a possible explanation for such phenomena. ${ }^{6,7}$ The electric field strength in the gap between closely-spaced particles is much larger than the applied field strength (potential difference between the electrodes divided by their separation). At such large field strengths, the fluid conductivity increases 
with field strength due to field-enhanced dissociation of species within the fluid. ${ }^{8}$ This causes the field strength between the particles to increase sublinearly with the applied field strength, eventually saturating at large field strengths. This results in the electrostatic stress scaling as $E^{n}$, where $n<2$, and the suspension conductivity increasing with field strength.

Attributing nonlinear conduction to field-enhanced dissociation in the bulk continuous phase has been questioned. , $^{3,9,10,11}$ Field-enhanced dissociation is limited, and likely cannot account for the leakage currents observed experimentally. ${ }^{11}$ Furthermore, dissociation in the bulk fluid becomes less important as particle size decreases, yet nonlinear conduction does not appear to be a function of particle size. ${ }^{10}$ Charge injection at the electrode/liquid or particle/liquid interfaces are alternative mechanisms for increased conductance. ${ }^{10}$ In this article, the particle/liquid and electrode/liquid interfaces are modified to examine these alternative mechanisms of nonlinear ER phenomena.

\section{Experimental}

Barium titanate $\left(\mathrm{BaTiO}_{3}\right.$, Aldrich, $99.9 \%$ pure) was ground and sifted to obtain particles with diameters in the range 53-90 $\mu \mathrm{m}$. Silicone oil (SF96, General Electric, $0.0968 \mathrm{~Pa} \cdot \mathrm{s}$ ) was dried for more than 1 week over molecular sieves [Aldrich, 4-8 mesh beads dried for $7 \mathrm{~h}$ under vacuum (-10 psig) at $185{ }^{\circ} \mathrm{C}$ prior to use]. The following materials were used as received: ethanol, acetic acid, toluene, octadecanol (each from Aldrich), R-trimethoxysilanes (United Chemical Technologies), Kraton D-1101 G rubber (Shell), and silicone rubber GI-1110 (Silicones, Inc.).

Barium titanate particles were dried under vacuum (-10 psig) at $110{ }^{\circ} \mathrm{C}$ for 2 days prior to suspension preparation. Suspensions were prepared by first weighing a quantity of oil and placing it in a vial. The appropriate amount of particles was added to the vial. The vial was then sealed and vigorously shaken.

Metal oxide particles exposed to air posses hydroxyl groups on their surfaces. ${ }^{12}$ Surface reactions were performed on some of the $\mathrm{BaTiO}_{3}$ particles to replace the surface hydroxyls with other groups. One reaction was a condensation that attached aliphatic $\left(\mathrm{C}_{18}\right)$ chains to the particle surface, ${ }^{13}$

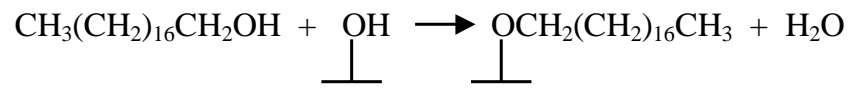

Particles were added to an octadecanol/ethanol solution. The ethanol was boiled off, and the remaining mixture refluxed at $200{ }^{\circ} \mathrm{C}$ for $4 \mathrm{~h}$. The mixture was then vacuum filtered to separate the particles, which were dried as described above.

The other reactions were alkoxysilane condensations, in which particle surface hydroxyls were reacted with R-trimethoxysilane, ${ }^{14}$

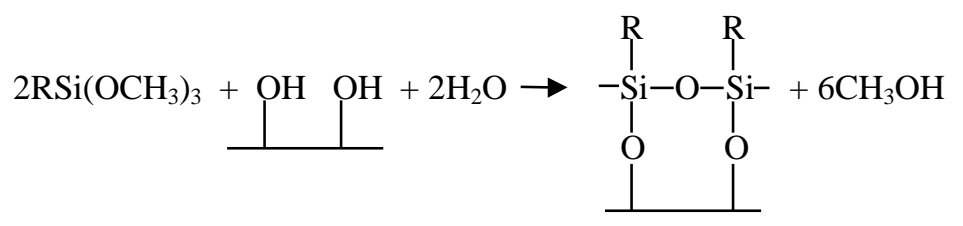


"R" groups employed in these reactions were:

$$
\begin{aligned}
& \text { "A" : }-n \mathrm{C}_{18} \mathrm{H}_{37}, \\
& \text { "B" : }-\left(\mathrm{CH}_{2}\right)_{3} \mathrm{NH}\left(\mathrm{CH}_{2}\right)_{2}\left(\mathrm{NH}_{2}^{+} \mathrm{Cl}^{-}\right) \mathrm{CH}_{2}\left(\mathrm{C}_{6} \mathrm{H}_{4}\right) \mathrm{CHCH}_{2}, \\
& \text { "C" : }-\left(\mathrm{CH}_{2}\right)_{3}\left(\mathrm{NHC}_{2} \mathrm{H}_{4}\right)_{2} \mathrm{NH}_{2} .
\end{aligned}
$$

The trimethoxysilane was mixed with a $95 \%$ aqueous ethanol solution (adjusted with acetic acid to $4.5 \leq \mathrm{pH} \leq 5.5$ ) to yield a 2 weight\% final concentration. Particles were then added to the solution while stirring. After approximately 10 min, the particles were filtered and dried at $110{ }^{\circ} \mathrm{C}$ for $10 \mathrm{~min}$.

"Wet" particles were prepared by mixing particles in a vial with water. The particle/water suspension was stored under vacuum $\left(-10 \mathrm{psig} ; 23^{\circ} \mathrm{C}\right)$ for at least 2 days, until the excess water had evaporated.

To investigate the role of charge injection at electrode surfaces, electrodes were coated with polymer layers of different thicknesses. Two polymers were used: Kraton and silicone rubber. Kraton dissolved in toluene was spread over the electrodes and exposed to air to allow the toluene to evaporate. This process was repeated as necessary to obtain the desired layer thicknesses. The silicone rubber was prepared by vigorously mixing base and activator $(\approx 10: 1$ weight ratio). The solution was spread over the electrodes to the desired layer thicknesses and allowed to cure for at least 12 hours. The polymer film thicknesses were measured with a caliper after rheological and electrical measurements were completed.

Rheological measurements were made with a Bohlin VOR rheometer using parallel plates, modified for the application of large electric fields. ${ }^{15}$ Potential differences were produced by a Stanford Research Systems (model DS345) function generator and amplified by a Trek (model 10/10) amplifier.

Dynamic yield stress measurements consisted of the following steps. The suspension was placed between the parallel plates, which were separated by $1 \mathrm{~mm}$. The suspension was initially sheared $\left(\dot{\gamma}>10^{-1}\right)$ at zero field for $1 \mathrm{~min}$ to ensure uniform particle distribution. The desired field was then applied for $1 \mathrm{~min}$ without shear. Shear stresses were measured as a function of decreasing shear rate $(0.002$ $\left.\leq \dot{\gamma} \leq 3 \mathrm{~s}^{-1}\right)$. The dynamic yield stress was determined by extrapolating the shear stress-shear rate data to zero shear rate.

One method of probing nonlinear conduction is to measure the harmonics of the current passing through the suspension. ${ }^{3}$ Consider the application of a sinusoidal electric field, $E(t)=E_{1} \sin \omega t$, to a suspension. If conduction is linear, the current passing through the suspension will also be sinusoidal,

$$
I^{l i n .}(t)=I_{1} \sin \left(\omega t+\delta_{1}\right) .
$$

The primary phase angle, $\delta_{1}$, arises from the suspension capacitance. If conduction is nonlinear, the current will be a superposition of many sinusoidal functions, ${ }^{3}$

$$
I^{\text {nonlin. }}(t)=I_{1} \sin \left(\omega t+\delta_{1}\right)+I_{3} \sin \left(3 \omega t+\delta_{3}\right)+I_{5} \sin \left(5 \omega t+\delta_{5}\right)+\ldots
$$

The quantities $I_{k}(\mathrm{k}=1,3,5, \ldots)$ are harmonics of the current. The current harmonics and corresponding phase angles $\left(\delta_{1}, \delta_{3}, \delta_{5}, \ldots\right)$ provide insight into the kinetics of nonlinear conduction. The current harmonic measurement technique has been discussed previously. ${ }^{3}$ 

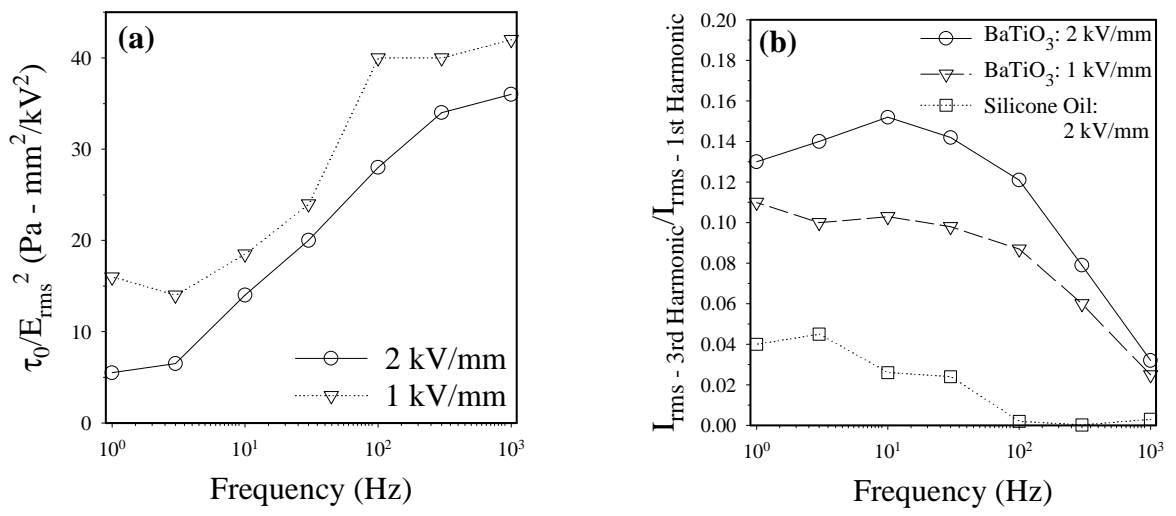

Figure 1. (a) $\tau_{o} / E_{r m s}^{2}$ as a function of frequency for a 10 vol\% suspension of untreated $\mathrm{BaTiO}_{3}$ particles in silicone oil at 1 and $2 \mathrm{kV} / \mathrm{mm}$. (b) Third harmonic of the current (relative to the fundamental harmonic) through a $10 \mathrm{vol} \%$ suspension of untreated $\mathrm{BaTiO}_{3}$ particles in silicone oil at 1 and $2 \mathrm{kV} / \mathrm{mm}$ and through silicone oil at $2 \mathrm{kV} / \mathrm{mm}$ as functions of frequency.

\section{Results}

Barium Titanate Suspensions. The dynamic yield stress scaled by $E_{r m s}^{2}$ at 1 and $2 \mathrm{kV} / \mathrm{mm}$ is plotted as a function of electric field frequency for a $10 \mathrm{vol} \%$ suspension of untreated barium titanate particles in silicone oil in Fig. 1a. If the suspension's ER response were linear, the data at different field strengths would collapse onto a single curve. The data do not superpose; the scaled yield stress is larger at $1 \mathrm{kV} / \mathrm{mm}$ than at $2 \mathrm{kV} / \mathrm{mm}$, with the yield stress proportional to $E_{r m s}^{n}$, where $n$ varies with frequency over the range $0.5 \leq n \leq 1.8$.

This rheological data differs from that shown previously, ${ }^{3}$ where the scaled yield stresses superposed for $\omega / 2 \pi \geq 100 \mathrm{~Hz}$. We believe this discrepancy is a result of batch-to-batch differences in the dielectric and electrical properties of $\mathrm{BaTiO}_{3}$ particles. All of the particles used for the data in this report came from the same batch.

The third current harmonic relative to the fundamental harmonic is plotted as a function of frequency for a $10 \mathrm{vol} \%$ suspension of untreated barium titanate particles in silicone oil at 1 and $2 \mathrm{kV} / \mathrm{mm}$ and for silicone oil at $2 \mathrm{kV} / \mathrm{mm}$ in Fig. 1b. The harmonic ratios decrease with frequency and increase with field strength, as expected for suspensions whose conductivities increase with field strength. The third harmonics for the suspension were at least three times larger than those for silicone oil. The primary phase angle, $\delta_{1}$, varies only slightly with field strength.

Modified Particle/Liquid Interfaces. The scaled yield stresses for $10 \mathrm{vol} \%$ suspensions of treated $\mathrm{BaTiO}_{3}$ particles in silicone oil at 1 and $2 \mathrm{kV} / \mathrm{mm}$ are plotted as functions of frequency in Fig. 2. The data at different field strengths for the treated particle suspensions are similar to that of the untreated particle suspension, in that they do not superpose. For each suspension, $\tau_{o} \propto E_{r m s}^{n}$, where $n$ varies with frequency over the range $0.5 \leq n \leq 1.9$. Modifying the particle/liquid interface 

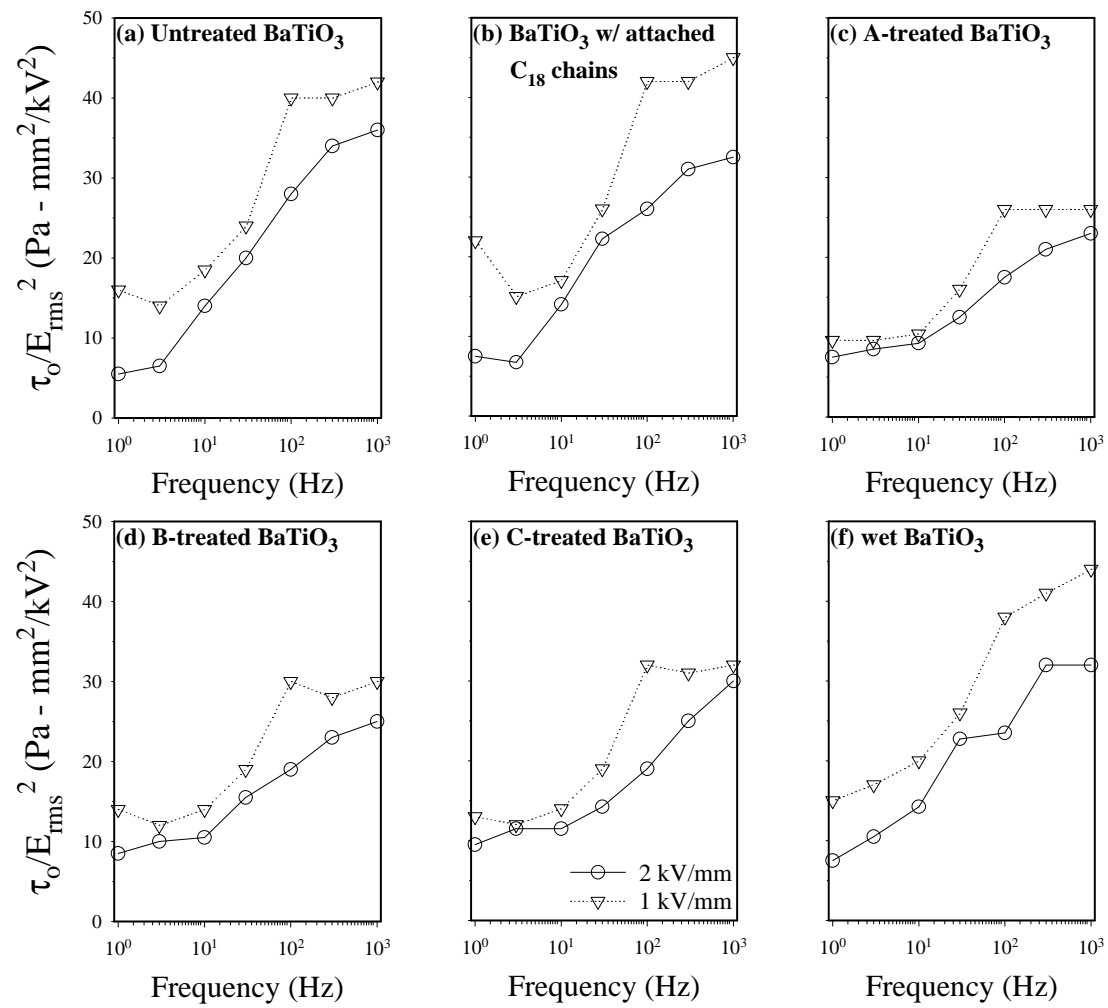

Figure 2. $\tau_{o} / E_{r m s}^{2}$ as functions of frequency for $10 \mathrm{vol} \%$ suspensions of (a) untreated $\mathrm{BaTiO}_{3}$ (b) $\mathrm{BaTiO}_{3}$ with attached $\mathrm{C}_{18}$ chains, (c) A-treated $\mathrm{BaTiO}_{3}$, (d) B-treated $\mathrm{BaTiO}_{3}$, (e) C-treated $\mathrm{BaTiO}_{3}$, and (f) wet $\mathrm{BaTiO}_{3}$ particles in silicone oil at 1 and $2 \mathrm{kV} / \mathrm{mm}$.

did not significantly affect the nonlinear electrorheological response.

The third current harmonics relative to the fundamental harmonics are plotted as functions of electric field frequency for $10 \mathrm{vol} \%$ suspensions of treated barium titanate particles in silicone oil at $2 \mathrm{kV} / \mathrm{mm}$ in Fig. 3. The harmonic ratios of the treated $\mathrm{BaTiO}_{3}$ suspensions are qualitatively similar to those measured for untreated $\mathrm{BaTiO}_{3}$ suspensions. The primary phase angles differ only slightly from those measured for the untreated suspensions. Again, this indicates that the surface modifications did not significantly alter the character of nonlinear conduction in these suspensions.

Modified Electrode/Liquid Interfaces. The scaled yield stresses are plotted as functions of frequency for $10 \mathrm{vol} \%$ suspensions of untreated $\mathrm{BaTiO}_{3}$ particles in silicone oil at 1 and $2 \mathrm{kV} / \mathrm{mm}$ in Fig. 4 for bare electrodes and for sets of two Kraton-coated electrodes. The two coatings differ in their thicknesses. Kraton 1 has layer thicknesses of 10 and $30 \mu \mathrm{m}$ for the top and bottom electrodes, respectively, and Kraton 2 has thicknesses of 250 and $200 \mu \mathrm{m}$. Coating the electrodes altered the nonlinear rheological responses. The yield stresses scaled as $\tau_{o} \propto E_{r m s}^{n}$ with $n$ 


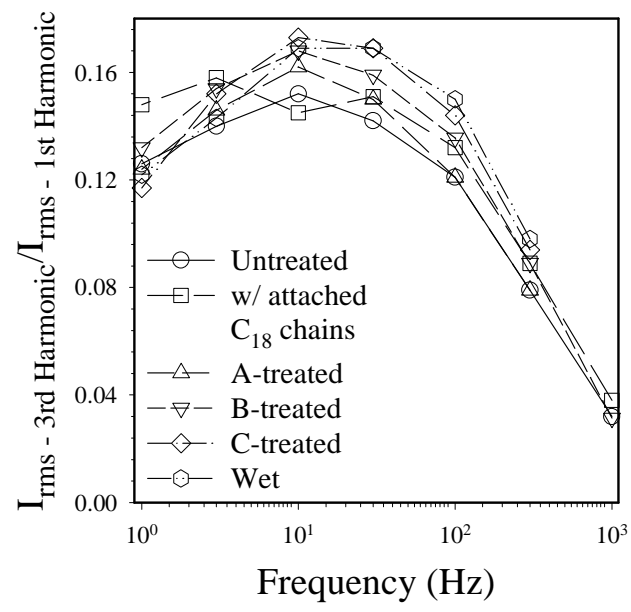

Figure 3. Third current harmonic (relative to the fundamental harmonic) as a function of frequency through 10 vol\% suspensions of untreated $\mathrm{BaTiO}_{3}, \mathrm{BaTiO}_{3}$ with attached $\mathrm{C}_{18}$ chains, $\mathrm{A}$, $\mathrm{B}$, and C-treated $\mathrm{BaTiO}_{3}$, and wet $\mathrm{BaTiO}_{3}$ particles in silicone oil at $2 \mathrm{kV} / \mathrm{mm}$.
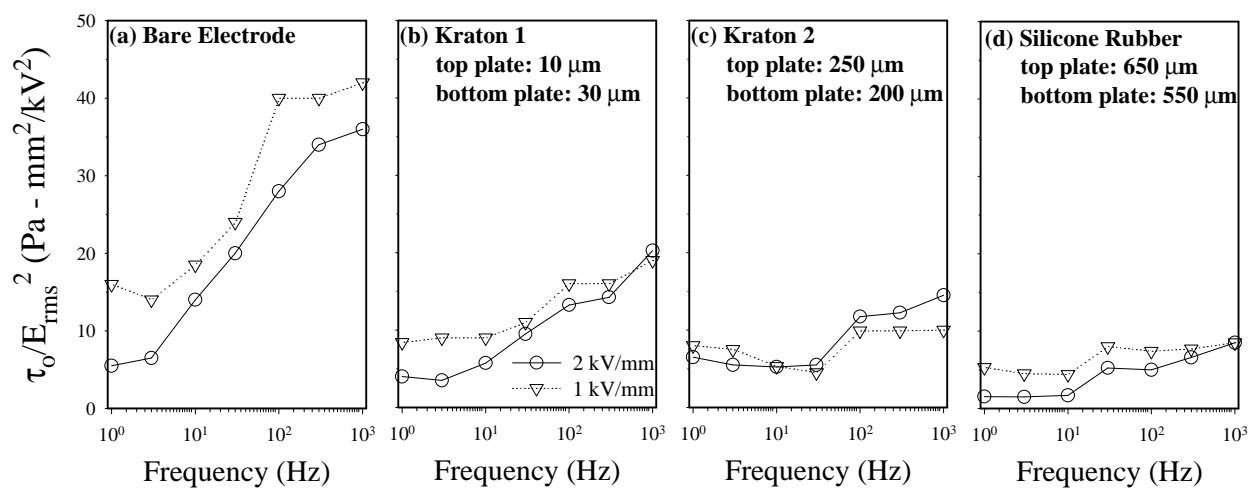

Figure 4. $\tau_{o} / E_{r m s}^{2}$ as functions of frequency for $10 \mathrm{vol} \%$ suspensions of untreated $\mathrm{BaTiO}_{3}$ particles in silicone oil at 1 and $2 \mathrm{kV} / \mathrm{mm}$. The yield stresses were measured with bare electrodes, two types of Kraton-coated electrodes, and silicone rubber-coated electrodes. The coatings differed in their thicknesses. The Kraton 1 coated electrodes had layer thicknesses of 10 and $30 \mu \mathrm{m}$ for the top and bottom plates, respectively, the Kraton 2 coated electrodes had thicknesses of 250 and $200 \mu \mathrm{m}$, and the silicone rubber-coated electrodes had thicknesses of 650 and $550 \mu \mathrm{m}$.

increasing with frequency, and $n \geq 2$ at some frequencies with the coated electrodes. In contrast, $n<2$ at all frequencies with bare electrodes. The layer thickness also affects the field-dependence. With the thicker Kraton 2 coated electrodes, $1.5 \leq n$ $\leq 2.5$, compared to the thinner Kraton 1 coated electrodes where $0.7 \leq n \leq 2.1$.

The type of polymer plays a significant role in altering the ER response. The scaled yield stress measured with silicone rubber-coated electrodes is plotted as 
a function of frequency for $10 \mathrm{vol} \%$ suspensions of untreated $\mathrm{BaTiO}_{3}$ particles in silicone oil at 1 and $2 \mathrm{kV} / \mathrm{mm}$ in Fig. $5 \mathrm{~d}$. The silicone rubber-coated electrodes had thicknesses of 650 and $550 \mu \mathrm{m}$ for the top and bottom plates, respectively. Although the silicone rubber coatings were more than twice as thick as the Kraton coatings, the yield stresses for the silicone rubber-coated plates had a weaker field dependence $(0.25 \leq n \leq 2)$. Also, the scaled yield stresses were smaller for the silicone rubber-coated electrodes than for the Kraton-coated electrodes.

Coating the electrodes also diminished the measured yield stresses, with the scaled yield stress decreasing with increasing coating thickness. There are several possible explanations. Because the impedance of the polymer coating is greater than that of the suspension, the field strength within the suspension will be smaller than the applied field strength. At large frequencies, we may estimate the field strength reduction in the suspension. Assuming relative dielectric constants of 8 and 3 for the suspension and polymer coatings, respectively, the field strength is reduced by approximately $10 \%, 34 \%$, and $52 \%$ for the Kraton 1, Kraton 2, and silicone rubber-coated systems, respectively. This could account for a significant fraction of the reduction in yield stress illustrated in Fig. 4.

Another possible explanation is that the friction force exerted by the particulate chains on the electrodes is reduced. The polymer coatings increase the separation between the electrodes and the particles, and thus decrease their electrostatic attraction. The normal force, and therefore the static and sliding friction forces, exerted by the chains on the electrodes are therefore reduced. The chains may then slide more easily over the electrodes, resulting in a smaller yield stress.

The third current harmonics relative to the fundamental harmonics are plotted as functions of electric field frequency for $10 \mathrm{vol} \%$ suspensions of untreated barium titanate particles in silicone oil between bare and coated electrodes at $2 \mathrm{kV} / \mathrm{mm}$ in Fig. 5. The harmonic ratios for the coated electrodes are smaller than those for bare electrodes at all frequencies. The harmonic ratio also decreased with electrode coating thickness. This indicates that modifying the electrode interface alters nonlinear conduction in these suspensions, and suggests that nonlinear conduction is intimately related to charge injection at the electrode/liquid interface.

\section{Conclusion}

Barium titanate suspensions show a nonlinear ER response. The dynamic yield stresses scale as $E^{n}$, with $n<2$, indicative of nonlinear conduction. The current also has significant harmonic content which increases with field strength, consistent with a suspension conductivity that increases with field strength.

Modifying the particle/liquid interfaces does not change the nonlinear behavior. Modifying the electrode/liquid interface, however, significantly changes the electrorheological response. With polymer coated electrodes, the field-dependence of the yield stress was altered. This change in the nonlinear ER response was accompanied by a decrease in the current harmonic content. These features are consistent with a decrease in the nonlinear content of the conduction in the suspensions. It appears, therefore, that nonlinear conduction in ER suspensions is intimately related to charge injection at the electrode/liquid interface.

ERconf99: submitted to World Scientific on February 11, 2002 


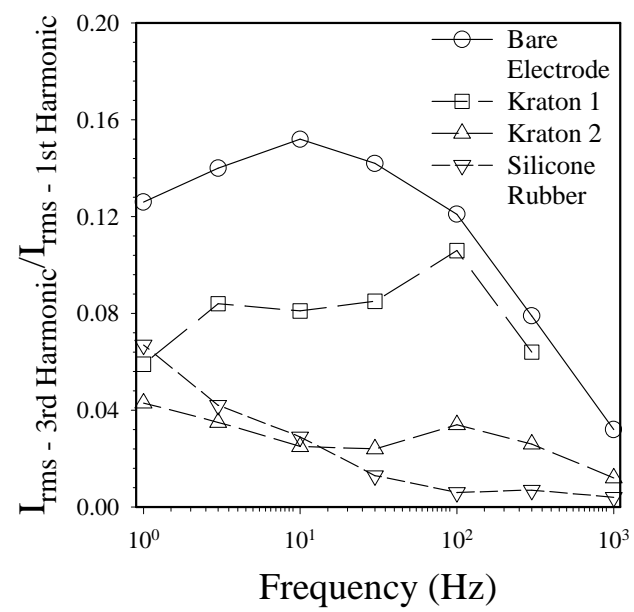

Figure 5. Third current harmonic (relative to the fundamental harmonic) as functions of frequency through 10 vol\% suspensions of untreated $\mathrm{BaTiO}_{3}$ particles in silicone oil between bare, Kraton 1 coated, Kraton 2 coated, and silicone rubber-coated electrodes at $2 \mathrm{kV} / \mathrm{mm}$.

\section{References}

1. W.M. Winslow, J. Appl. Phys. 2051137 (1949).

2. M. Parthasarathy and D.J. Klingenberg, Mater. Sci. Eng. R17 57 (1996).

3. P.J. Rankin and D.J. Klingenberg, J. Rheol. 42639 (1998).

4. L.C. Davis, J.Appl. Phys. 811985 (1997).

5. L.C. Davis and J.M. Ginder in Progress in Electrorheology, ed. K.O. Havelka and F.E. Filisko (Plenum, New York, 1995), 107.

6. N. Felici, J.N. Foulc, and P. Atten in Proceedings of the 4th International Conference of Electrorheological Fluids, ed. R. Tao (World Scientific, Singapore, 1994), 139.

7. J.N. Foulc, P. Atten, and N. Felici, J. Electrostat. 39103 (1994).

8. L. Onsager, J. Chem. Phys. 2599 (1934).

9. P. Atten, J.N. Foulc, and H. Benqassmi in Progress in Electrorheology, ed. K.O. Havelka and F.E. Filisko (Plenum, New York, 1995), 231.

10. C. Boissy, P. Atten, J.N. Foulc, Int. J. Mod. Phys. B 102991 (1996).

11. N.J. Felici, J. Elect. 40567 (1997).

12. R.J. Hunter Foundations of Colloid Science, Vol I, (Clarendon, Oxford, 1989).

13. L. Marshall and C.F. Zukoski, J. Phys. Chem. 941164 (1990).

14. Silicon Compounds: Register and Review (United Chemical Tech., Inc., 1997).

15. Y.D. Kim Ph.D. thesis (University of Wisconsin, Madison, WI, 1996).

\section{Acknowledgements}

This work was supported in part by the National Science Foundation (CTS-9502276) 